\title{
Derivatives of Sodium Boranocarbonate as Novel CO-Releasing Molecules (CO-RMs)
}

\author{
Tamil S. Pitchumony ${ }^{\S a}$, Bernhard Spingler ${ }^{\mathrm{a}}$, Roberto Motterlini ${ }^{\mathrm{b}}$, and Roger Alberto*a \\ §SCS Poster Prize Winner
}

\begin{abstract}
Despite the apparent and well-known toxic effects of carbon monoxide (CO), studies on the intriguing biological roles of this molecule are rapidly emerging. Recent investigations have brought to the limelight various physiological effects of $\mathrm{CO}$ which include, among others, vasorelaxation and inhibition of organ rejection after transplantation. The importance of CO in biology can be compared with another gas, nitric oxide (NO), an essential and ubiquitous signalling molecule. This parallelism led to the tantalising concept of using $\mathrm{CO}$ for therapeutic purposes wherein compounds that transport and deliver this gas to a target tissue would clearly facilitate both the clinical feasibility and the specificity of $\mathrm{CO}$ therapy. Sodium boranocarbonate, $\mathrm{Na}_{2} \mathrm{H}_{3} \mathrm{BCO} \mathrm{C}_{2} \mathrm{H}$ (1) was the first water-soluble and non-transition metal containing CO-releasing molecule (CO-RM) to be identified. In order to tune the rate of $\mathrm{CO}$ release, we modified 1 and synthesised and characterized various derivatives which release $\mathrm{CO}$ under physiological conditions but at rates different from the parent compound 1. The synthesis, structure and CO-releasing properties of ester and amide derivatives of 1 will be presented together with some biological studies carried out with a selection of the compounds.
\end{abstract}

Keywords: Carbon monoxide $\cdot$ CO-releasing molecules

\section{Introduction}

Some twenty years ago, the reputation of nitric oxide (NO) started to shift from a noxious, polluting gas to a vital molecule as a messenger in processes essential for almost all living organisms including humans. ${ }^{[1,2]}$ As a consequence, in 1992 the prestigious journal Science proclaimed NO 'molecule of the year'. In retrospective, it does not come as a surprise that in 1998 the

${ }^{*}$ Correspondence: Prof. Dr. R. Alberto ${ }^{a}$

Tel.: + 41446354631

Fax: + 41446356803

E-mail: ariel@aci.uzh.ch

aAnorganisch-chemisches Institut

Universität Zürich

Winterthurerstrasse 190

$\mathrm{CH}-8057$ Zürich

bVascular Biology Unit

Department of Surgical Research

Northwick Park Institute for Medical Research

Harrow, Middlesex, UK intense research around the biological and medicinal importance of NO culminated with the Nobel Prize in Medicine awarded to scientists who discovered its physiological function.

The situation is distinctly different in the case of carbon monoxide (CO). The toxic effect of $\mathrm{CO}$ gas is coherently typified at biochemical levels by the high affinity for hemoglobin and cytochromes, which are both heme-dependent proteins essential for life. Only about ten years ago it became clear that $\mathrm{CO}$ possesses, like NO, versatile properties as a signalling mediator. ${ }^{[3,4]}$ Beside being a potent vasodilator, $\mathrm{CO}$ can exert anti-inflammatory, anti-apoptotic and anti-proliferative effects to name just a few of its pharmacological actions (Fig. 1). ${ }^{[5]}$ With the emerging evidence that small amounts of $\mathrm{CO}$ produced by the organism is a key element in life processes, scientists began to search for compounds capable of releasing $\mathrm{CO}$ at specific sites with a controllable and tuneable rate. ${ }^{[6]}$ Compared to NO-releasing drugs, the task of developing $\mathrm{CO}$ carriers as pharmaceuticals is much more challenging since $\mathrm{CO}$ is a very stable molecule which does not readily and reversibly react with substrates but needs to be incorporated in usually strong additional bonds. The availability of 'CO-releasing molecules' (CO-RMs) that are compatible with a physiological environment not only provides excellent perspectives for biological or medicinal purposes but has also a great impact on organometallic chemistry. The immediate choice for compounds to be utilized as CO-RMs is carbonyl-complexes, which biochemical properties and biological effects have been studied but there are concerns about their solubility, stability and the fact that they contain transition metals which may or may not come as disadvantages. ${ }^{[6-8]}$

In the course of our studies for the aqueous syntheses of ${ }^{99 m}$ Tc complexes, we have found that sodium boranocarbonate, $\mathrm{Na}\left[\mathrm{H}_{3} \mathrm{BCO}_{2} \mathrm{H}\right](\mathbf{1})$ releases $\mathrm{CO}$ with a rate that is $\mathrm{pH}-$ and temperature-dependent. ${ }^{[9]}$ This makes it a suitable molecule to carry 'bound' $\mathrm{CO}$ to target organs and to release $\mathrm{CO}$ slowly. To further influence the rate of $\mathrm{CO}$ release, we have now prepared various amide and ester derivatives of $\mathbf{1}$. These derivatives can be considered as 'pro-drugs' of CO-RMs whilst having the potential to carry the molecule to specific sites in cells or organisms. ${ }^{[10]}$

\section{Results and Discussion}

The basic structure and some of the prepared derivatives are presented in the Scheme. The $\mathrm{p} K_{\mathrm{a}}$ values of $\mathbf{1}$ are compara- 


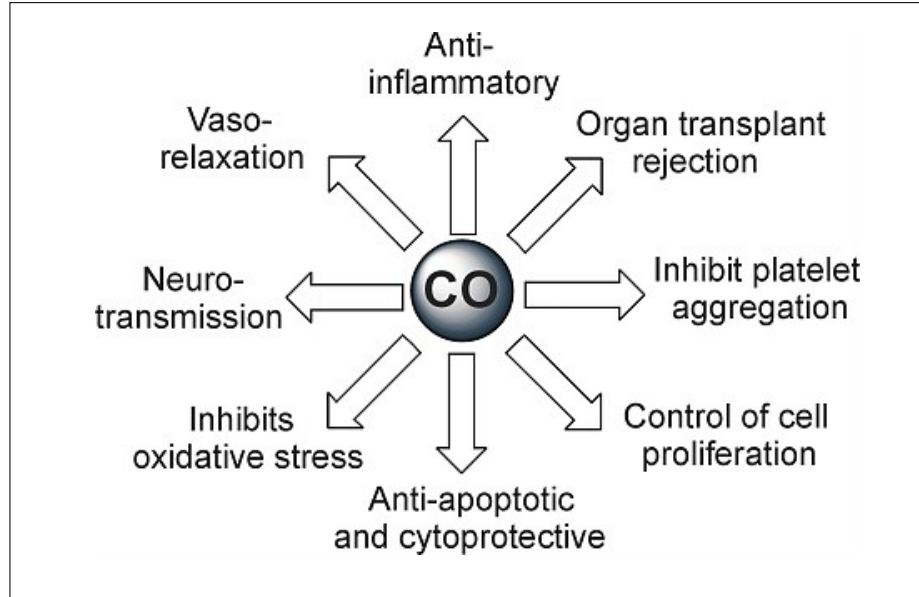

Fig. 1. The multiple pharmacological effects of $\mathrm{CO}$ as a signalling mediator in living systems

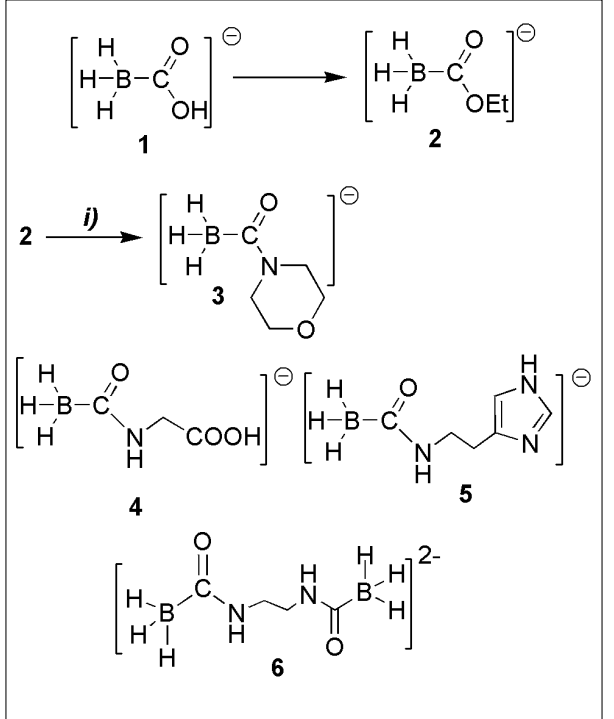

Scheme. Pathway to 'activated' ester 2 and subsequent reaction i) with amines leading to the corresponding amides 3-6 of 1
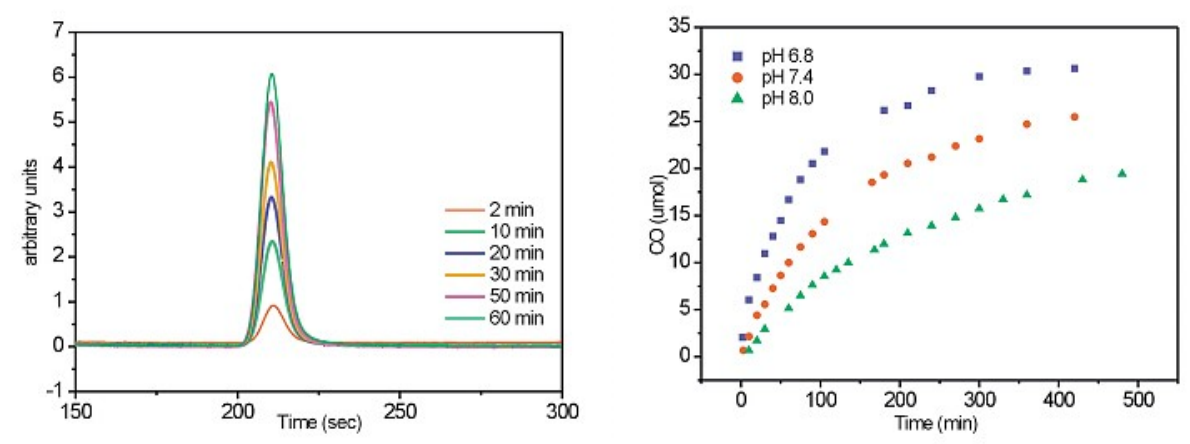

Fig. 3. $\mathrm{CO}$ release as a function of time for $\mathrm{Na}\left[\mathrm{H}_{3} \mathrm{BCO}(\mathrm{gly})\right]$ (4) at $\mathrm{pH}$ 6.8. The amount of $\mathrm{CO}$ was determined by GC-TCD

ble to carbonic acid (hence the name). The second $\mathrm{p} K_{\mathrm{a}}$ is difficult to determine since protonation of 1 leads to irreversible cleavage of water and subsequent $\mathrm{CO}$ release. The derivatization of $\mathbf{1}$ is also difficult, since the hydrides, despite being bound rather stably, are somewhat prone to hydrolysis or sub-

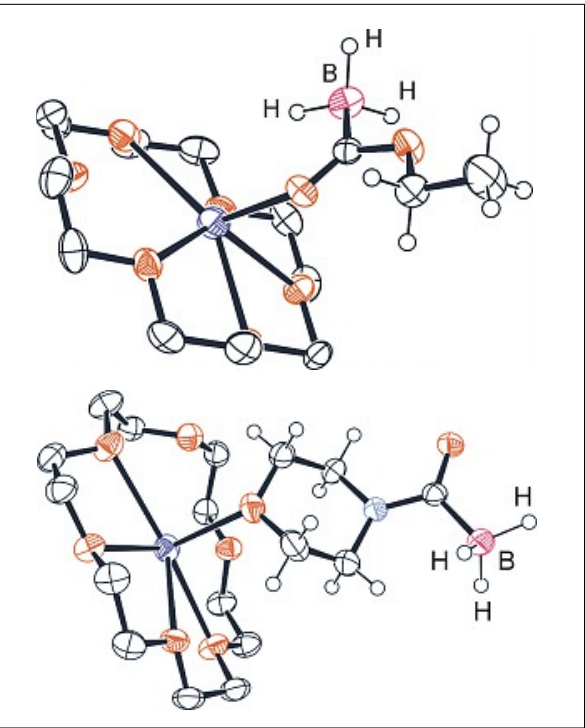

Fig. 2. X-ray structures of the starting material 2 and the corresponding morpholino-amide $\mathbf{3}$ only possible when encapsulating the $\mathrm{Na}^{+}$ in 18-crown-6. Although esters are usually only reacting very slowly with amines, we found that $\mathbf{2}$ reacted at a reasonable rate with primary and secondary amines to form the corresponding amides. Along this synthetic route, we have prepared the amides with morpholine $\mathrm{Na}\left[\mathrm{H}_{3} \mathrm{BCO}\right.$ (morph)] (3), glycine $\mathrm{Na}\left[\mathrm{H}_{3} \mathrm{BCO}(\right.$ gly)] (4), histamine $\mathrm{Na}\left[\mathrm{H}_{3} \mathrm{BCO}\right.$ (hist) $]$ (5) and $\mathrm{Na}_{2}\left[\left\{\mathrm{H}_{3} \mathrm{BCO}\right\}_{2}(\mathrm{en})\right](\mathbf{6})$ with ethylene-diamine containing two molecules of $\mathbf{1}$ bound to each terminal amine. A number of further derivatives could be prepared implying that the strategy is general and can also be applied to other potential linking groups. All compounds are stable white solids which could be fully characterized, some of them by X-ray structure analysis.

In an earlier work, we studied the rate of $\mathrm{CO}$ release of 1 under physiological conditions and at $37^{\circ} \mathrm{C}$ by means of a myoglobin $(\mathrm{Mb})$ assay. ${ }^{[10]}$ Upon binding of $\mathrm{CO}$ to $\mathrm{Mb}$, the spectrum changes substantially and rates can be determined. We found that the rate of $\mathrm{CO}$ release showed a very steep $\mathrm{pH}$ dependence with $\mathrm{t}_{1 / 2}$ being $2.5 \mathrm{~min}$ at $\mathrm{pH}=$ $5.5,7.1 \mathrm{~min}$ at $\mathrm{pH}=7.0$ and $21 \mathrm{~min}$ at $\mathrm{pH}$ $=7.4$, respectively. We studied $\mathrm{CO}$ release with the newly prepared amide derivatives by a gas chromatograph equipped with thermal conductivity detector (GC-TCD) and by ${ }^{11} \mathrm{~B}$ and ${ }^{1} \mathrm{H}$ NMR experiments. Since the release of $\mathrm{CO}$ requests two consecutive steps, (first hydrolysis of the amide to $\mathbf{1}$ and then conversion of $\mathbf{1}$ to $\mathrm{CO}$ and some borate products), we expected a decrease of the overall rate. A typical time course of the amount of $\mathrm{CO}$ as a function of time determined by GC-TCD for compound 4 is shown in Fig. 3. It is obvious that the strong $\mathrm{pH}$-dependence is maintained. The observed $\mathrm{t}_{1 / 2}$ are now approximately $60 \mathrm{~min}$ at $\mathrm{pH}=6.8,150 \mathrm{~min}$ at $\mathrm{pH}=7.4$ and 400 $\min$ at $\mathrm{pH}=8.0$, respectively. The corresponding time courses for compounds 3-6 are very comparable and do not seem to be significantly dependent on the nature of the amide. It is clear that the elucidation of the mechanism has to give an insight in the different mechanistic steps but the qualitative numbers given above clearly demonstrate the delay in CO release.

We would like to emphasize the difference between amides of carboxylic acids, which essentially do not hydrolyse under physiological conditions. The peptide bond is extremely stable whereas the amides 3-6 decompose on a comparably short time scale. This might point to a mechanism different from acid-catalysed amide bond cleavage.

Besides the quantification of released $\mathrm{CO}$, decomposition can also be followed by ${ }^{1} \mathrm{H}$ and ${ }^{11} \mathrm{~B}$ NMR spectroscopy. During the reaction, the ${ }^{11} \mathrm{~B}$ signal of $\mathbf{3}$ and of $\mathbf{1}$ are the only sharp signals to be observed by NMR. as ethanol overnight led to almost quantitative yield to the corresponding ethylester $\mathrm{Na}\left[\mathrm{H}_{3} \mathrm{BCO}_{2} \mathrm{Et}\right]$ (2) (Scheme), whose structure could be confirmed by $\mathrm{X}$-ray analysis (Fig. 2). As encountered with all presented compounds, crystallization was 




Fig. $4 .{ }^{11} \mathrm{~B}$ NMR of the hydrolysis of $\mathbf{3} \rightarrow \mathbf{1}$ (left) and the corresponding ${ }^{1} \mathrm{H}$ spectrum in the $\mathrm{B}-\mathrm{H}$ region (right)

All subsequent decomposition products display only very broad unresolved bumps, probably due to the presence of ill-defined borates and/or borato-hydrides. ${ }^{11} \mathrm{~B}$ NMR spectra are given in Fig. 4. After 2 min, the two signals of $\mathbf{1}$ and $\mathbf{3}$ reach a certain ratio which is kept constant up to $24 \mathrm{~h}$.

The same observations can be made in the ${ }^{1} \mathrm{H}$ NMR spectra. All compounds comprising the ' $\mathrm{H}_{3} \mathrm{~B}$ ' moiety showed a nice quartet centred around $0.6 \mathrm{ppm}$. The conversion of $\mathbf{3} \rightarrow \mathbf{1}$ resulted in two clearly distinguishable sets of signals, indicative for $\mathbf{1}$ and $\mathbf{3}$ and decreasing with the same rate the corresponding signals in the ${ }^{11} \mathrm{~B}$ NMR spectra. A corresponding spectrum is also shown in Fig. 4.

According to our experimental results and at a first glance, the mechanism of $\mathrm{CO}$ release consists in amide hydrolysis and subsequent protonation of $\mathbf{1}$ with release of water to form highly reactive $\mathrm{H}_{3} \mathrm{BCO}$, which then decomposes to $\mathrm{CO}$ and boratohydrides. The deduction of single rate constants from kinetic measurements is currently under investigation. Preliminary results indicate that the mechanism does not consist of irreversible forward steps only but that $\mathrm{H}_{3} \mathrm{BCO}$ in particular may hydrolyse back to 1 with a rate comparable to its decomposition. Unambiguous elucidation of the kinetic results is under way.

In conclusion, we have shown that the half-life of $\mathrm{CO}$ release of the newly prepared derivatives of boranocarbonate 1 can be fine-tuned by the introduction of functionalities at the carboxylic group. This also implies that the model amines selected for this study can be replaced by some metabolically active targeting functions allowing the CO-RM to be channelled to specific sites in living organism where it needs to exert its action. It can also be assumed that these derivatives not only find applications as CO-RMs in biology but also in other areas such as the synthesis of carbonyl complexes directly from water.

\section{Acknowledgments}

The authors thank the University of Zurich and hemoCORM Ltd. UK for financial support.

Received: February 25, 2008

[1] M. A. Gillman, F. J. Lichtigfeld, Nature 1994, 367, 28.

[2] A. R. Butler, D. L. H. Williams, Chem. Soc. Rev. 1993, 22, 233.

[3] R. Motterlini, B. E. Mann, T. R. Johnson, J. E. Clark, R. Foresti, C. J. Green, Curr. Pharm. Design 2003, 9, 2525.

[4] T. R. Johnson, B. E. Mann, J. E. Clark, R. Foresti, C. J. Green, R. Motterlini, Angew. Chem., Int. Ed. 2003, 42, 3722.

[5] T. Morita, M. A. Perrella, M. E. Lee, S. Kourembanas, Proc. Natl. Acad. Sci. USA 1995, 92, 1475.

[6] R. Motterlini, J. E. Clark, R. Foresti, P. Sarathchandra, B. E. Mann, C. J. Green, Circ. Res. 2002, 90, E17.

[7] J. E. Clark, P. Naughton, S. Shurey, C. J. Green, T. R. Johnson, B. E. Mann, R. Foresti, R. Motterlini, Circ. Res. 2003, 93, E2.

[8] R. Foresti, J. Hammad, J. E. Clark, T. R. Johnson, B. E. Mann, A. Friebe, C. J. Green, R. Motterlini, Brit. J. Pharmacol. 2004, 142, 453 .

[9] R. Alberto, K. Ortner, N. Wheatley, R. Schibli, A. P. Schubiger, J. Am. Chem. Soc. 2001, 123, 3135.

[10] R. Motterlini, P. Sawle, S. Bains, J. Hammad, R. Alberto, R. Foresti, C. J. Green, Faseb. J. 2004, 18. 